\title{
CAPTISOL® AS CHIRAL SELECTOR IN CAPILLARY ELECTROPHORESIS OF NON-ACIDIC DRUGS
}

\author{
MONICA BUDÄU', GABRIEL HANCU ${ }^{\prime *}$, ZOLTÁN ISTVÁN SZABÓ2, HAJNAL KELEMEN ${ }^{1}$, AURA RUSU', \\ DANIELA LUCIA MUNTEAN", ANCA GABRIELA CÂRJE
}

\author{
${ }^{I}$ Department of Pharmaceutical Chemistry, Faculty of Pharmacy, University of Medicine and Pharmacy, Târgu Mureș, Romania; \\ ${ }^{2}$ Department of Pharmaceutical Industry and Pharmaceutical Management, Faculty of Pharmacy, \\ University of Medicine and Pharmacy, Târgu Mureş, Romania; \\ ${ }^{3}$ Department of Analytical Chemistry and Drug Analysis, Faculty of Pharmacy, University of Medicine and Pharmacy, Târgu Mureş, Romania;
}

\section{ABSTRACT}

Enantioseparation of nine extensively used chiral pharmaceutical substances from different pharmacological classes and with different structural characteristics has been investigated by capillary zone electrophoresis using Captisol ${ }^{\circledR}$ (sulfobuthylether- $\beta$-cyclodextrin sodium salt) as chiral selector. The influence on the chiral separation of several parameters including $\mathrm{pH}$ and concentration of the background electrolyte, chiral selector concentration, applied voltage, system temperature and injection parameters were studied and optimized in order to obtain increased chiral resolution and shorten analysis time. The results were compared with those obtained with native and derivatized neutral cyclodextrins ( $\beta$-cyclodextrin and hydroxypropryl- $\beta$-cyclodextrin). All model molecules were resolved using Captisol ${ }^{R}$ as chiral additive and optimized electrophoretic conditions, proving the efficiency of this anionic derivative of $\beta$ - cyclodextrin as chiral selector in capillary electrophoresis.

Keywords: chiral separation, Captisol®, capillary electrophoresis, cyclodextrines, pharmaceutical analysis

\section{INTRODUCTION}

Almost half of the drugs currently used in therapy have a chiral center in the molecule, but only approximately $25 \%$ of them are administered in the form of a pure enantiomer. It is well known that usually the aimed pharmacological effect is restricted to one of the enantiomers, called eutomer; while the other enantiomer, called distomer can be inactice, less active or sometimes can cause unwanted adverse effects. Enantiomers differ in terms of absorption, distribution, metabolic pathway or binding affinity to proteins and receptors. ${ }^{1}$

Chiral drugs are generally synthesized as racemic mixtures or as single enantiomers; analysis of a pharmaceutical form comprising of a single enantiomer requiring sometimes determination of very low concentrations of enantiomer impurity levels of less than $1 \%$. Quantification of these concentration levels is often difficult and challenging for the analyst, especially when good selectivity and high sensitivity is required. The most frequently used separation technique in the enantioseparation of pharmaceutical substances is without doubt high performance liquid chromatography (HPLC), but in recent years capillary electrophoresis (CE) is gaining more and more followers in modern chiral analysis. ${ }^{2}$

The advantages of CE compared to conventional HPLC methods in the enantioseparation of chiral substances are being related to the: rapid and simple method development, small amounts of solvent, sample and chiral selector required for the separation and especially with the high selectivity in choosing and changing the chiral selector. Also in CE, usually a direct method of separation is used, by simply adding the chiral selector in the background electrolyte (BGE). ${ }^{2,3,4}$

In order to obtain chiral separation, the enantiomers have to come into contact with a chiral environment and to form two different diastereomeric complexes. According to the rule of three points of interaction by Dlagliesh, chiral recognition depends on at least three simultaneous interactions between selector and chiral molecule and at least one of these interactions needs to be stereoselective in order to allow enantiomer discrimination. ${ }^{2}$

Cyclodextrines (CDs) are by far the most frequently used chiral additives in $\mathrm{CE}$; in modern chiral analysis a large number of native and derivatized, neutral and ionized CD derivatives being used as chiral selectors. CDs mechanism as chiral selector involves a complexation through inclusion of the hydrophobic part of the analyte in the hydrophobic cavity of the CD. The stability of the formed complex is influenced by several parameters, including chemica structure and hydrophobicity of the analyte, $\mathrm{CD}$ type and concentration, background electrolyte (BGE) composition, buffer $\mathrm{pH}$ and temperature. ${ }^{5,6}$

The utility of native CDs as chiral additives is sometimes limited, and therefore a large number of derivatized CDs have been developed and introduced as chiral selectors. By derivatization, hydrophobicity and charge can be altered and affect, electrophoretic mobility or complexing ability of the analytes. Enantioseparation by $\mathrm{CE}$ requires that either the analyte or the used $\mathrm{CD}$ is electrically charged.,4,7
The use of charged CDs enables additional electrostatic interactions between analyte and the chiral selector, which can significantly influence the resolution of the enantiomers. Charged CDs can be employed for the chira separation of both charged and uncharged analytes, because, in the ionized state, this type of CDs migrates with their own electrophoretic mobility. Further advantages of charged CD derivatives are related to the better solubility and the ability to display additional electrostatic interactions. , $^{5,8}$

Captisol $\AA$ is a particular type of sulfobutyl ether derivative of $\beta-C D$ with a range of six to seven sulfobutyl ether groups per CD molecule (figure 1). Because of the very low pKa of the sulfonic acid groups, Captisol ${ }^{\circledR}$ carries multiple negative charges at physiologically compatible $\mathrm{pH}$ values. The fourcarbon butyl chain coupled with repulsion of the end group negative charges allows for an "extension" of the CD cavity. This often results in stronger binding to drug candidates than can not be achieved using other modified CDs. It also provides a potential for ionic charge interactions between the $\mathrm{CD}$ and a positively charged drug molecule. Captisol ${ }^{\circledR}$ was rationally designed in order to maximize safety and optimize interaction to improve the solubility, stability and administration of active ingredients..$^{9,10,11}$

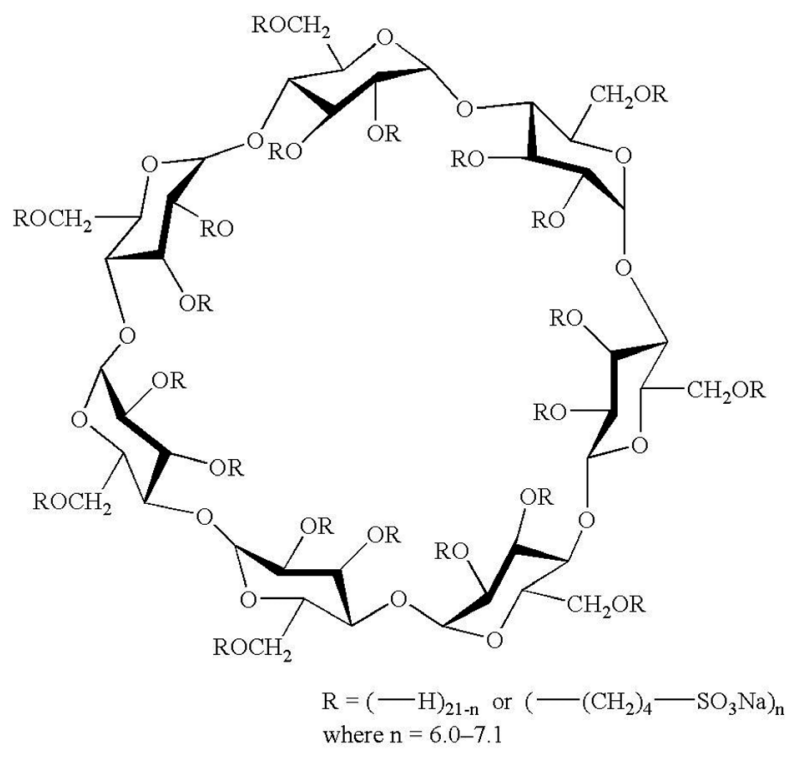

Sulfobutyl Ether- $\beta$-Cyclodextrin (Captisol $\otimes)$

Figure 1: Captisol ${ }^{\circledR}$ (sulfobutyl ether $\beta$-cyclodextrin sodium salt) chemical structure. 
Sulfobuthylether- $\beta$-cyclodextrins (SBE- $\beta$-CDs) are anionic derivatives of $\beta-\mathrm{CD}$ that has proven during the years to be successful in separating a large variety of enantiomeric compounds. Usually SBE- $\beta$-CDs used as chiral selectors have an average degree of substitution of four and a substitution range from one to ten. Previous works with SBE- $\beta-C D$ has shown that several compounds exhibited peak tailing and loss of efficiency due to the electrodispersion phenomenon. ${ }^{12,13,14,15}$

The first application of SBE- $\beta-C D$ as chiral selector in CE was described 20 years ago for the separation of ephedrine, pseudoephedrine and several structurally related compounds ${ }^{12}$; and since then different types of SBE- $\beta$-CD with specific degrees of substitution have been used for the chiral discrimination of basic drugs ${ }^{14,15}$; as a recent example Captisol® was applied successfully for the separation of 16 benzofurys and other psychoactive compounds used on the illicit market as recreational drugs in an aqueous ammonium acetate solution containing acetonitrile. ${ }^{16}$

In the present investigation, a number of nine optically active pharmaceutical substances were selected as model compounds to assess the utility of Captisol ${ }^{\circledR}$ as chiral selector. These compounds represent excellent examples of the need for chiral specific determination procedures because of the stereoselectivity of their pharmacological activities. Different compounds from different therapeutic classes were selected due to their great prevalence in therapy and also taking in consideration their particular structural and stereochemical characteristics. As model molecules we selected $\beta$-adrenergic receptor blockers (carvedilol, propranolol, sotalol), H1 antihistamines (cetirizine, promethazine), dihydropyridine calcium channel blocker (amlodipine), selective serotonin reuptake inhibitor (fluoxetine), diuretic (indapamide), opioid analgesic (tramadol).

\section{EXPERIMENTAL}

\section{Apparatus}

Analyses were performed using an Agilent $7100 \mathrm{CE}$ system (Agilent, Waldbronn, Germany) equipped with a diode-array-detector (DAD). For the separation we used silica-fused capillaries (Agilent, Waldbronn, Germany) with a total length of $48.5 \mathrm{~cm}$ (effective length of $40 \mathrm{~cm}$ ) and an internal diameter of $50 \mu \mathrm{m}$. The data was processed using Chemstation 7.01 (Agilent, Germany) software. The $\mathrm{pH}$ of the buffer solutions was measured with a Terminal 740 $\mathrm{pH}$-meter (Inolab, Germany). The UV spectrum of the studied substances was recorded with a Specord 210 spectrophotometer (Analytik Jena, Germany).

\section{Chemicals and reagents}

The racemic mixtures of the selected chiral compounds were obtained from various sources: R,S-amlodipine besiltate (Fako Ilaclari A.S, Istanbul, Turkey), R,S-carvedilol (Sun Pharmaceutical Industries, Mumbai, India), R,Scetirizine hydrochloride (Tonira Pharma Ltd., Guajarat, India), R,S-fluoxtine hydrochloride (Mantena Laboratories Ltd, Hyderabad, India), R,S-indapamide (Moehs Productos Quimicos, Barcelona, Spain), R,S-promethazine hydrochloride (Morepen Laboratories Ltd., New Delhi, India), R,S-propranolol hydrochloride (Moehs Productos Quimicos, Barcelona, Spain), R,S-sotalol hydrochloride (Moehs Productos Quimicos, Barcelona, Spain), R,S-tramadol hydrochloride (Sun Pharmaceutical Industries, Mumbai, India). For four of the studied chiral substances we had at our disposal also pure enantiomers: S-carvedilol (Sun Pharmaceutical Industries, Mumbai, India), R-cetirizine hydrochloride (Tonira Pharma Ltd., Guajarat, India), R-propranolol (Moehs Productos Quimicos, Barcelona, Spain), S-sotalol hydrochloride (Moehs Productos Quimicos, Barcelona, Spain). All of the studied substances were of pharmaceutical grade.

Captisol ${ }^{\circledR}$ (SBE- $\beta-C D$ sodium salt, research grade, average degree of substitution: 6.5) was obtained from Cydex Pharmaceuticals Inc. (La Jolla, CA, USA). The neutral CDs ( $\beta-\mathrm{CD}$ and HP- $\beta-C D)$ were obtained from Cyclolab (Budapest, Hungary).

The following reagents of analytical grade were used: phosphoric acid (Chimopar, Bucharest, Romania), methanol, sodium hydroxide (Lach Ner, Neratovice, Czech Republic), sodium tetraborate, disodium hydrogenophosphate, sodium didydrogenophosphate (Merck, Darmstadt Germany). Purified water was prepared using a Milli-Q Plus water purification system (Millipore, Milford, MA, USA)

Sample peparation

Sample stock solutions were prepared by dissolving the substances in methanol in a concentration of $100 \mu \mathrm{g} / \mathrm{mL}$ and later diluted to the appropriate concentration. The samples were introduced in the system at the anodic end of the capillary by hydrodynamic injection. All samples and buffers were filtered through a $0.45 \mu \mathrm{m}$ pore size Cellulose filter and degassed for 5 minutes using an ultrasonic bath.

\section{Electrophoretic procedure}

Every time before starting the experiments, the capillary was conditioned by flushing 10 minutes with water, 15 minutes with $0.1 \mathrm{M} \mathrm{NaOH}$ and 5 minutes with buffer electrolyte. The capillary was rinsed for 1 minute with $0.1 \mathrm{M}$ sodium hydroxide and buffer solutions before each electrophoretic determination.

In the preliminary analysis we applied some "standard" electrophoretic conditions for a $\mathrm{CE}$ analysis: temperature $20^{\circ} \mathrm{C}$, applied voltage $+25 \mathrm{kV}$, injection pressure/time $50 \mathrm{mbar} / 3 \mathrm{sec}$, sample concentration $10 \mu \mathrm{g} / \mathrm{ml}$.

UV-spectra of the analytes were recorded in a range of 200 and $400 \mathrm{~nm}$ in order to determine the optimum detection wavelength of each compound.

\section{RESULTS AND DISCUSSION} 2.

The chemical structures of the selected substances are presented in figure

Taking in consideration the $\mathrm{pKa}$ values and the structural characteristics of the selected substances we can conclude that the large majority of the selected substances exhibit basic characteristics, excepting cetirizine which is a zwitterionic molecule.

To find the suitable conditions for the chiral separation of the model molecules, a series of preliminary experiments were conducted with different buffer compositions at different $\mathrm{pH}$ values, in order to characterize the electrophoretic behavior of the analytes in an achiral system over a wide adjustable $\mathrm{pH}$ range (2-11). In the preliminary analysis we used: $25 \mathrm{mM}$ phosphoric acid ( $\mathrm{pH}-2.1), 25 \mathrm{mM}$ sodium didydrogenophosphate $(\mathrm{pH}-5.0)$, $25 \mathrm{mM}$ disodium hydrogenophosphate - sodium didydrogenophosphate (1:1) $(\mathrm{pH}-7.0)$ and $25 \mathrm{mM}$ sodium tetraborate $(\mathrm{pH}-9.3)$ BGEs respectively and we modified the $\mathrm{pH}$ of the buffer by adding a $0.1 \mathrm{M}$ sodium hydroxide solution.

Afterwards, the CDs were added in the buffer solutions, and the electrophoretic behavior of the analytes in a chiral system was observed and characterized over the same $\mathrm{pH}$ range. In the screening process initial concentration of $10 \mathrm{mM}$ for the neutral CDs and $5 \mathrm{mM}$ for SBE- $\beta-C D$ were used.

Buffer $\mathrm{pH}$ is an important condition in $\mathrm{CE}$, as the degree of dissociation of the charged selector, analyte charge, and the electroosmotic flow (EOF) are all affected by $\mathrm{pH}{ }^{19,20}$ When $\mathrm{pH}$ increased, the velocity of the EOF increased and migration times were shorter; as it is well known that EOF will increase considerably with increasing $\mathrm{pH}$.

While $\beta-C D$ and HP- $\beta-C D$ are not charged, an advantage of using SBE$\beta-C D$ as chiral selector is that it maintains its charge over the entire $\mathrm{pH}$ range useful in $\mathrm{CE}$, as the presence of the sulfonic group makes the $\mathrm{CD}$ derivative negatively charged over the studied $\mathrm{pH}$ range. ${ }^{13,14}$ Using an acidic BGE, Captisol ${ }^{\circledR}$ migrates towards the anode and the enantiomers of the basic drugs migrates towards the cathode, while EOF is close to zero; resulting in very long migration times for the studied analytes and weak chiral interactions.

At acidic $\mathrm{pH}$ values the weak EOF cannot compensate for the negative electrophoretic mobility of the anionic charged $\mathrm{CD}$, which interacts strongly with the basic compounds, delaying their migration towards the cathode.

Captisol ${ }^{\circledR}$ proved to be an efficient chiral selector when using BGE with $\mathrm{pH}$ values above 5.0; as at $\mathrm{pH}$ values above 5.0 are favored ion pairing interaction between the positively charged analytes and the anionic $\mathrm{CD}$, thus, transporting the analyte towards the anode.

An increase in the buffer concentration, increases ionic strength and decreases EOF, affecting electrostatic interactions between Captisol $\AA$ and analytes, resulting in increased analysis time and improved peak shapes. However the increase of the buffer concentration is limited by band broadening due to Joule effect caused by increased current; consequently in the current study we used buffer concentrations of $25 \mathrm{mM}$.

Compared with neutral CDs the effect of the concentration of charged CDs on the selectivity of the enantioseparation is more pronounced. ${ }^{19,20} \mathrm{We}$ investigated experimentally the optimum concentration of Captisol ${ }^{\circledR}$ from 1 to $10 \mathrm{mM}$; as higher concentration generated high currents which led to the instability of the electrophoretic system. For the neutral CD we investigated the effect of CD concentration on an interval between 5 and $25 \mathrm{mM}$. An increase of Captisol ${ }^{\circledR}$ concentration increased also analysis time of the analytes, probably because changes in the viscosity of the buffer, and changes in EOF due to increased ionic strength. Also increasing the $\mathrm{CD}$ concentration leads to an increase of the migration time of the analytes (with detection at the cathode) because they are transported to the anode due to complexation by the negatively charged CE. The optimum Captisol ${ }^{\circledR}$ concentration was set at $5 \mathrm{mM}$.

Addition of an organic modifier such as methanol or acetonitrile to the buffer resulted only in longer migration times as there was no significant 
improvement in the chiral separation.

Running voltage did not have a strong effect on the resolution; while a decrease in temperature led to extension in analysis time and to an increase of the chiral resolution. An equilibrium between applied voltage and system temperature should be established, in order to obtain an adequate resolution of the separation and a satisfactory analysis time. Taking in consideration these aspects we used a voltage of $+25 \mathrm{kV}$ at a temperature of $15^{\circ} \mathrm{C}$

The injection parameters (injection pressure and time) had an important effect on the peaks shape and amplitude; in order to achieve a quantifiable signal/noise ratio and to avoid band broadening we chose a high injection pressure (50 mbar) and a short injection time (1 second).<smiles>COC(=O)C1=C(C)NC(C)=C(C(=O)OCCOCCN)C1c1ccccc1Cl</smiles><smiles>[R10]C([R20])[C@@H](CCOCC(=O)O)N1CCN(C(c2ccccc2)c2ccc(Cl)cc2)CC1</smiles><smiles>COc1ccccc1OCCNCC(O)COc1cccc2[nH]c3ccccc3c12</smiles><smiles>CNCCC(Oc1ccc(C(F)(F)F)cc1)c1ccccc1</smiles><smiles>CC1Cc2ccccc2N1NC(=O)c1ccc(Cl)c(S(=O)(=O)O[Ga])c1</smiles><smiles>CC(CN1c2ccccc2Sc2ccccc21)N(C)C</smiles>

Promethazine $\mathrm{pKa}-9.1$<smiles>CC(C)NCC(O)c1ccc(NS(=O)(=O)O[GaH2])cc1</smiles><smiles>C=CCC(=C)OCC(O)CNC(C)C</smiles>

\section{Propranolol} $\mathrm{pKa}-9.5$<smiles>COc1cccc(C2(O)CCCCC2CN(C)C)c1</smiles>

Figure 2: Chemical structures of the selected compounds. The chiral centers are marked with an asterix. ${ }^{17,18}$ 
Table 1 summarizes the optimum experimental conditions ( $\mathrm{CD}$ type and concentration, buffer $\mathrm{pH}$ ) and the results (migration times of the separated zones $-\mathrm{t}_{1}$ and $t_{2}$, separation factor $-\alpha$, resolution $-\mathrm{R}$ ) obtained for those compounds, which had stereoselective interactions.

Table 1: Capillary electrophoretic chiral separation data of model compounds

\begin{tabular}{|c|c|c|c|c|c|c|c|}
\hline Chiral compound & Chiral selector & CD concentration $[\mathrm{mM}]$ & pH & $t_{1}(\min )$ & $\mathbf{t}_{2}$ (min) & $\mathbf{R}$ & $\alpha$ \\
\hline \multirow{3}{*}{ Amlodipine } & $\beta-C D$ & 15 & 2.50 & 6.30 & 6.45 & 0.86 & 1.02 \\
\hline & $\mathrm{HP}-\beta-\mathrm{CD}$ & 20 & 2.50 & 4.50 & 4.90 & 2.17 & 1.08 \\
\hline & SBE- $\beta-C D$ & 5 & 7.00 & 11.40 & 12.30 & 2.79 & 1.08 \\
\hline \multirow{3}{*}{ Carvedilol } & $\beta-C D$ & 10 & 2.50 & 11.00 & 11.50 & 2.74 & 1.04 \\
\hline & $\mathrm{HP}-\beta-\mathrm{CD}$ & 20 & 2.50 & 12.90 & 13.50 & 2.54 & 1.04 \\
\hline & SBE- $\beta-C D$ & 5 & 9.30 & 5.80 & 6.20 & 1.93 & 1.05 \\
\hline \multirow{3}{*}{ Cetirizine } & $\beta-\mathrm{CD}$ & - & - & - & - & - & - \\
\hline & $\mathrm{HP}-\beta-\mathrm{CD}$ & - & - & - & - & - & - \\
\hline & SBE- $\beta-C D$ & 5 & 7.00 & 6.40 & 7.00 & 2.54 & 1.08 \\
\hline \multirow{3}{*}{ Fluoxetine } & $\beta-C D$ & - & - & - & - & - & - \\
\hline & $\mathrm{HP}-\beta-\mathrm{CD}$ & - & - & - & - & - & - \\
\hline & SBE- $\beta-C D$ & 5 & 9,30 & 6.20 & 6.30 & 0.85 & 1.01 \\
\hline \multirow{3}{*}{ Indapamide } & $\beta-C D$ & - & - & - & - & - & - \\
\hline & $\mathrm{HP}-\beta-\mathrm{CD}$ & - & - & - & - & - & - \\
\hline & SBE- $\beta-C D$ & 5 & 7.00 & 5.70 & 6.30 & 4.30 & 1.10 \\
\hline \multirow{3}{*}{ Promethazine } & $\beta-C D$ & 10 & 2.50 & 10.50 & 10.70 & 0.88 & 1.02 \\
\hline & $\mathrm{HP}-\beta-\mathrm{CD}$ & - & - & - & - & - & - \\
\hline & SBE- $\beta-C D$ & 5 & 7.00 & 9.70 & 10.10 & 1.55 & 1.04 \\
\hline \multirow{3}{*}{ Propranolol } & $\beta-\mathrm{CD}$ & 10 & 2.50 & 9.40 & 9.80 & 1.13 & 1.04 \\
\hline & $\mathrm{HP}-\beta-\mathrm{CD}$ & 10 & 2.50 & 11.00 & 11.50 & 1.46 & 1.04 \\
\hline & SBE- $\beta-C D$ & 5 & 9.30 & 4.80 & 5.00 & 1.23 & 1.04 \\
\hline \multirow{3}{*}{ Sotalol } & $\beta-C D$ & - & - & - & - & - & - \\
\hline & HP- $\beta-C D$ & 20 & 2.50 & 9.30 & 9.70 & 0.93 & 1.03 \\
\hline & SBE- $\beta-C D$ & 5 & 9.30 & 4.20 & 4.45 & 1.53 & 1.05 \\
\hline \multirow{3}{*}{ Tramadol } & $\beta-\mathrm{CD}$ & - & - & - & - & - & - \\
\hline & $\mathrm{HP}-\beta-\mathrm{CD}$ & - & - & - & - & - & - \\
\hline & SBE- $\beta-C D$ & 5 & 9.30 & 3.95 & 4.20 & 1.52 & 1.06 \\
\hline
\end{tabular}

The separation factors $(\alpha)$ were calculated as the ratio of the migration times of the optical isomers, and the resolution (R) was obtained by the $\mathrm{R}=2\left(\mathrm{t}_{2}-\mathrm{t}_{1}\right) /\left(\mathrm{w}_{1}+\mathrm{w}_{2}\right)$ equation, where the migration times $\left(\mathrm{t}_{1}\right.$ and $\left.\mathrm{t}_{2}\right)$ and the peak-widths $\left(\mathrm{w}_{1}\right.$ and $\left.\mathrm{w}_{2}\right)$ were marked for the slow and fast migrating enantiomers, respectively. The separation factors and resolution parameters are characterizing the separation. A value above 1.04 for $\alpha$ and above 1.50 for $\mathrm{R}$ generally means baseline separation of the two enantiomers.

From the nine tested molecules, stereoselective interactions when using Captisol ${ }^{\circledR}$ as chiral selector were observed for all compounds, eight being baseline separated and for one (fluoxetine) we noticed only a slight peak splitting. In contrast no chiral resolution was obtained when using the two neutral CDs for four of the model compounds (cetirizine, fluoxetine, indapamide, tramadol), and for promethazine and sotalol only peak splitting was observed.

When using Captisol ${ }^{\circledR}$ at neutral and basic pH values of the BGE we obtained sistematically better chiral resolution and shorter migration times for the analytes in comparison with the results obtained with $\beta-\mathrm{CD}$ and $\mathrm{HP}-\beta-\mathrm{CD}$ respectively.

The electropherograms of the enantioseparations of the studied substances when using Captisol ${ }^{\circledR}$ as chiral selector are presented in figure 3.

Tramadol have two chiral centers, but actually is a mixture of only 2 of the theoretically possible stereoisomer, i.e. the $(1 \mathrm{R}, 2 \mathrm{R})$ and $(1 \mathrm{~S}, 2 \mathrm{~S})$ stereoisomers, which migrated always in two zones with approximately equal peak areas.

The migration order of the studied enantiomers was verified by analyzing the racemic mixtures with the pure enantiomers. We established the migration order for four of the studied substances using the optimized electrophoretic conditions presented before (table 2). Dependent on the interaction between $\mathrm{CD}$ and analyte, the enantiomer with the stronger affinity to the selector migrates slower. 

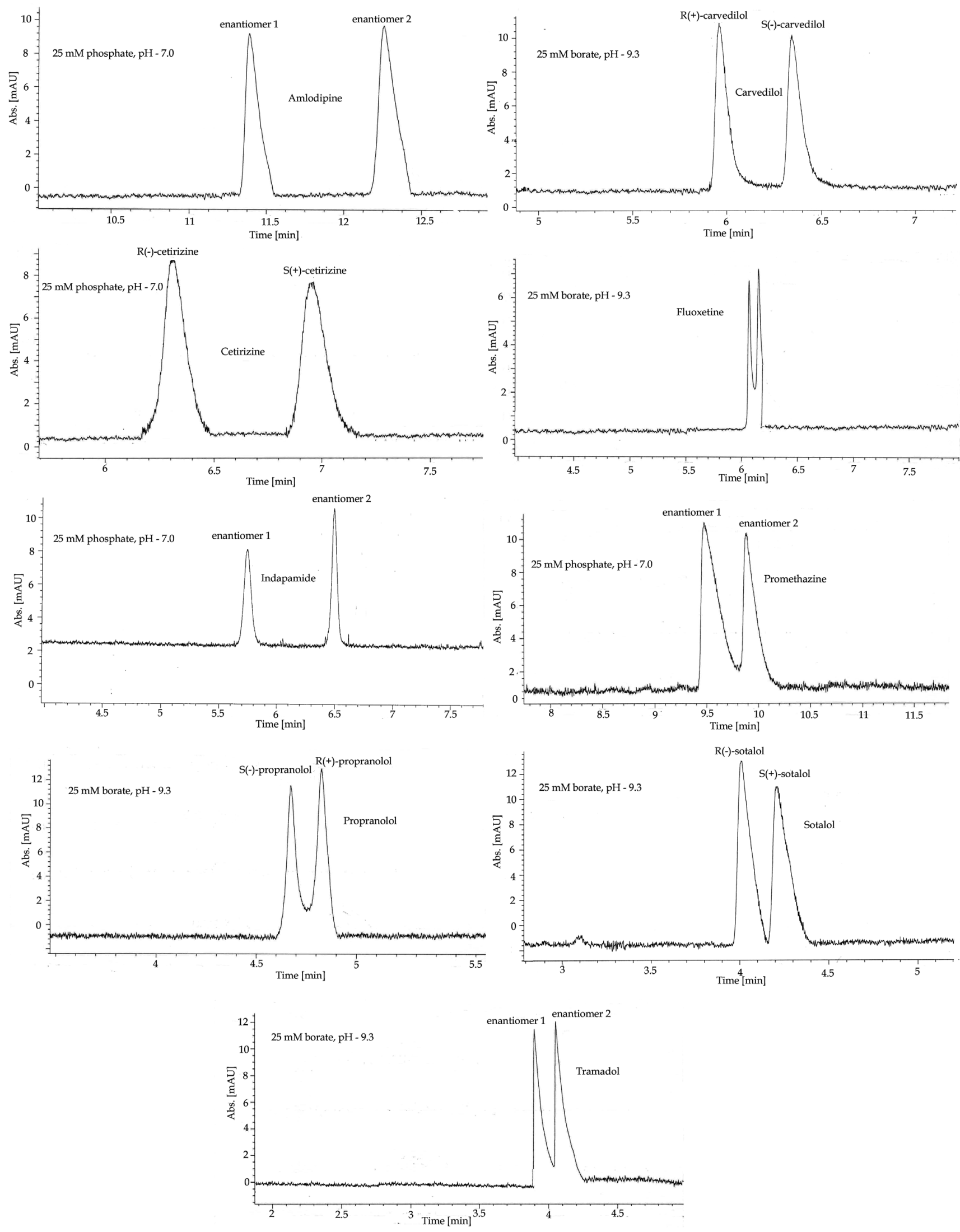

Figure 3: Chiral separation of the model compounds using Captisol ${ }^{\text {a }}$ as chiral selector (conditions: buffer concentration: $25 \mathrm{mM}$, Captisolconcentration: 5 $\mathrm{mM}$, applied voltage: $+25 \mathrm{kV}$, temperature: $15^{\circ} \mathrm{C}$, injection pressure/time: $50 \mathrm{mbar} / 1 \mathrm{sec}$.) 
Table 2 Migration order of enantiomers when using optimized separation conditions and Captisol ${ }^{\circledR}$ as chiral selector.

\begin{tabular}{|c|c|c|}
\hline Chiral compound & Enantiomer 1 & Enantiomer 2 \\
\hline Carvedilol & $\mathrm{R}(+)$-carvedilol & $\mathrm{S}(-)$-carvedilol \\
\hline Cetirizine & $\mathrm{R}(-)$-cetirizine & $\mathrm{S}(+)$-cetirizine \\
\hline Propranolol & $\mathrm{S}(-)$-propranolol & $\mathrm{R}(+)$-propranolol \\
\hline Sotalol & $\mathrm{R}(-)$-sotalol & $\mathrm{S}(+)$-sotalol \\
\hline
\end{tabular}

\section{CONCLUSIONS}

Captisol $\AA$ increase the mobility differences between effective mobility of the complexes formed between the enantiomers and the $\mathrm{CD}$, showed potential for electrostatic interactions that may help to stabilize complexes with analytes bearing opposite charges, which resulted in a wider separation window and potentially greater resolving power for the basic drugs. The sulfonic groups provide a $\mathrm{CD}$ derivative, which is anionic over the entire $\mathrm{pH}$ range accessible to $\mathrm{CE}$ experiments and which can thereby lead to ionic interaction in addition to the hydrophobic inclusion.

The optimal separation conditions for the the analytes were different in several cases; as there are three main aspects that should be considered in understanding and explaining the chiral separation process: the role of the chemical structure of the analyte, the effect of the experimental parameters and the structure of the chiral selector. The changes in the concentration of the chiral selector and in the $\mathrm{pH}$ of the BGE showed uneven effect on the resolution of the optical isomers. No direct correlation due to increase or decrease of the respective concentration or $\mathrm{pH}$ was noticed.

Captisol ${ }^{\circledR}$ can be successfully applied to the chiral separation of a large variety of optically active substances (amlodipine, carvedilol, cetirizine, indapamide, promethazine, propranolol, sotalol, tramadol), showing strong resolving power especially towards neutral and basic drugs while its selectivity for negatively charged analytes is poor. The presented methods may be used in routine control methods to assess the optical purity of different pharmaceutical substances with a relatively high chiral resolution and short analysis time.

\section{ACKNOWLEDGMENT}

Our work was supported with a project funded through Internal Research Grants by the University of Medicine and Pharmacy of Tîrgu Mureș, Romania (grant contract for execution of research projects nr. 22).

\section{REFERENCES}

1. L.A. Nguyen, H. He, C. Pham-Huy, Int. J. Biomed. Sci. 2, 85, (2006).

2. G. Gübitz, M.G. Schmid (eds.), Chiral Separations - Methods and Protocols. Humana Press, Totowa, New Jersey, 2004.

3. P. Schmitt-Kopplin (eds.), Capillary electrophoresis - Methods and Protocols. Humana Press, Totowa, New Jersey, 2008.

4. P. Mikus, Chiral Capillary Electrophoresis in current Pharmaceutical and Biomedical Analysis, InTech, Rijeka, 2012.

5. T. Schnitt, H. Engelhardt, Chromatographia 37, 475, (1993).

6. G. Gübitz, M.G. Schmid, J. Chromatogr. A 792, 179, (1997)

7. G. Gübitz, M.G. Schmid, J. Chromatogr. A 1204, 140, (2008).

8. L. Suntornsuk, Anal. Bioanal. Chem. 398, 29, (2010).

9. S. Sotthivirat, J.L. Haslam, V.J. Stella, Int. J. Pharm. 330, 73, (2007).

10. D.R. Luke, K. Tomaszewki, B. Damle, H.T. Schlamm, J. Pharm. Sci. 99, 3291, (2010).

11. A. Espada, M. Molina-Martin, Drug Discov. Today 17, 396, (2012).

12. R.J. Tait, D.O. Thompson, V.J. Stella, J.F. Stobaugh, Anal. Chem. 66, 4013, (1994).

13. C. Desidorio, S. Fanali, J. Chromatogr. A 716, 183, (1995).

14. D.J. Skanchy, G.H. Xie, R.J. Tait, E. Luna, C. Demarest, J.F. Stobaugh, Electrophoresis 20, 2638, (1999).

15. X. Ren, Y Dong, J. Liu, A. Huang, H. Liu, Y. Sun, Z. Sun, Chromatographia 50, 363, (1999).

16. M. Taschwer, M.G. Hofer, M.G. Schmid, Electrophoresis 35, 2793, (2014).

17. J:H. Block, J.M Beale (eds.), Wilson and Gisvold's textbook of Organic Medicinal and Pharmaceutical Chemistry. $12^{\text {th }}$ edition, Lippincott Williams\&Wilkins, Philadelphia, 2011.
18. Martindale, The Complete Drug Reference, $37^{\text {th }}$ edition, Pharmaceutical Press, London, 2011.

19. M. Dolezalova, S. Fanali, Electrophoresis 21, 3264, (2000).

20. G.S. Yang, D.M. Chen, Y. Yang, B. Tang, J.J. Gao, H.Y. Aboul-Enein, B. Koppenhoefer, Chromatographia 62, 441, (2005). 